\title{
GEOMETRICALLY NONLINEAR THEORY OF NATURALLY CURVED AND TWISTED RODS WITH FINITE ROTATIONS
}

\author{
By Masashi IURA* and Masaharu HIRASHIMA**
}

\begin{abstract}
The object of this paper is to develop a finite displacement theory of naturally curved and twisted rods undergoing finite rotations. Particular attention is paid to investigate the coupling of finite rotations in space under the Bernoulli-Euler hypothesis. A finite rotation vector is employed to derive the displacement field available for finite rotations. A new variable is introduced as a fourth parameter associated with rotations of cross sections. Then the twist and curvatures after the deformation are expressed in terms of four parameters without using small-strain assumptions. The equilibrium equations and the associated boundary conditions, in which second order terms with respect to displacement components are fully taken into account, are derived from the principle of virtual work. The accuracy of the present equilibrium equations are confirmed through comparisons with those obtained by the equilibrium method.
\end{abstract}

\section{INTRODUCTION}

The analysis of geometrically nonlinear behavior of thin rods, used as common structural elements, has received considerable attention of many investigators. It is widely accepted that a finite rotation plays an important role in the nonlinear behavior of rods. Since the finite rotations are not quantities in the vector space, the nonlinear behavior of rods with finite rotations in space has been studied by various approaches. In this paper, with the help of the finite rotation vector $\theta$, we develop a geometrically nonlinear theory of naturally curved and twisted rods by taking account of the coupling of finite rotations in space.

In a small displacement theory, the displacement field of rods has been derived frequently from the assumptions of strain field ${ }^{1)}$. This approach has been also available for a finite displacement theory ${ }^{2) \sim 4}$. However, if we utilize the above approach, we must solve the nonlinear partial differential equations for displacement components. Since most solutions of the nonlinear differential equations can not be obtained, some approximations are introduced at an early stage; that is, the second and higher order terms with respect to the axial displacement are neglected in the strain-displacement relations. Consequently, the coupling of finite rotations in space is not taken into account accurately. The resulting governing equations are available only for the second order theory of rods with small rotations ${ }^{5) 6}$.

In the case of planar nonlinear behavior of elastic rods with finite rotations, the coupling of finite rotations does not occur since there is only one degree of freedom for rotations. Nishino, Kurakata and Goto $^{7)}$ have developed a geometrically nonlinear theory of planar rods under the Bernoulli-Euler hypothesis, and pointed out that, if the energy method is used, the small strain assumptions must be

* Member of JSCE, Dr. Eng., Assistant Professor, Department of Civil and Structural Engineering, Tokyo Denki University (Hatoyama, Hiki-gun, Saitama)

** Member of JSCE, Dr. Eng., Professor, Department of Civil Engineering, Waseda University (Okubo 3-4-1, Shinjuku-ku, Tokyo) 
adopted after taking variations with respect to displacement components.

In the case of nonplanar nonlinear behavior of elastic rods, in which the coupling of finite rotations generally occur, various approaches exist for evaluating the finite rotations of rods. Maeda and Hayashi ${ }^{5}$ have used the finite rotation tensor as a measure for finite rotations. The accurate finite displacement field has been obtained by introducing three displacement and three rotation parameters. It is well known that four parameters are necessary and sufficient for the description of behavior of rods under the Bernoulli-Euler hypothesis. When the number of parameters decreases from six to four, Maeda and Hayashi have introduced the assumption of small rotations. Besseling ${ }^{8)}$ and Rosen and Friedmann ${ }^{9}$ have utilized the Euler angles as a measure for finite rotations. In these studies, also, the assumption of small rotations is employed at a stage of describing the nonlinear behavior of rods in terms of four parameters. Schroeder ${ }^{10)}$, Hirashima, Iura and Yoda ${ }^{11)}$, and Kotoguchi, Kubo and Nakai ${ }^{12)}$ have used the finite rotation vector $\omega$. Hirashima, Iura and Yoda have derived the more accurate displacement functions among these studies. However the inplane strains obtained from the displacement functions of Ref. (11) do not become zero. This is contradictory of the commonly used assumption that cross sections do not distort. Shimada ${ }^{13)}$, and Panayotounakos and Theocaris ${ }^{14)}$, using the equilibrium method, have derived the governing equations of tortuous rods. In these studies, the second order terms are neglected in the constitutive equations for twisting moment.

Kurakata $^{15)}$, Kurakata and $\mathrm{Nishino}^{6}$, and $\mathrm{Ai}$ and $\mathrm{Nishino}^{16)}$ have evaluated the finite rotations of nonplanar rods by using another approaches. They have pointed out that the fourth parameter, which corresponds to an angle of rotation of cross sections, can not be defined in the case of nonplanar finite displacement theory of thin-walled members ${ }^{15}$, and that the displacement components of beam axis are not always necessary to be chosen as independent parameters ${ }^{16}$. Finite rotations play an important role also in shell structures. Simmonds and Danielson ${ }^{17}$, Pietraszkiewic $z^{18)}$, and Iura and Hirashima ${ }^{19)}$ have developed a geometrically nonlinear shell theory by using the finite rotation vector $\boldsymbol{\Omega}$. Pietraszkiewicz and Badur ${ }^{20}$ have investigated the finite rotations in continuum mechanics with the use of the finite rotation tensor and the finite rotation vectors, $\omega, \Omega$ and $\theta$.

In this paper, utilizing the finite rotation vector $\theta$, we develop a geometrically nonlinear theory of naturally curved and twisted rods with finite rotations in space. In order to discuss the coupling of finite rotations in detail, we postulate that the warping of rods is neglected. The fundamental hypotheses employed here are itemized as follows :

(1) Cross sections of rods are constant along the beam axis.

(2) The beam axis before the deformation is a smooth space curve.

(3) The transveres plane is assumed to remain plane and normal to the beam axis throughout the deformation; that is, the Bernoulli-Euler hypothesis holds.

Throughout this paper, the summation rule is adopted and Latin indices will have the range 1, 2, 3.

\section{GEOMETRY BEFORE DEFORMATION}

Consider a naturally curved and twisted rod in a fixed Cartesian coordinates $X^{m}$ with the base vectors $\boldsymbol{i}_{m}$, as shown in Fig. 1. Two convected coordinate systems are introduced to describe the motion of the rod. One of these is the orthogonal curvilinear coordinates $\xi^{m}$ with the base vectors $\boldsymbol{e}_{m}$. The coordinate $\xi^{1}$ coincides with the beam axis at the point $\mathrm{S}$, while the coordinates $\xi^{2}$ and $\xi^{3}$ are taken along principal axes of the cross section of the rod. The other is the local Cartesian system of coordinates $Z^{m}$ with the base vectors $\boldsymbol{b}_{m}$. The coordinates $Z^{m}$ are chosen so that the base vectors $\boldsymbol{b}_{m}$ of $Z^{m}$ coincide with the base vectors $\boldsymbol{e}_{m}$ of $\xi^{m}$ at an arbitrary material point $\mathrm{P}$. The orientations of the present coordinate systems follow the right hand rule.

The position vector at the point $\mathrm{S}$ is given by

$x=x\left(\xi^{1}\right)=x^{m} i_{m}$. 
Then the tangent unit vector $\boldsymbol{e}_{1}$ in the reference state is obtained by

$$
e_{1}=x_{, 1}
$$

where ( ), denotes differentiation with respect to $\xi^{1}$. With the aid of the Frenet-Serret formulae, the following system of differential formulae holds:

$$
\left(\begin{array}{l}
e_{1,1} \\
e_{2,1} \\
e_{3,1}
\end{array}\right)=\left(\begin{array}{ccc}
0 & \varkappa_{3} & -\varkappa_{2} \\
-\varkappa_{3} & 0 & \varkappa_{1} \\
\varkappa_{2} & -\varkappa_{1} & 0
\end{array}\right)\left(\begin{array}{l}
e_{1} \\
e_{2} \\
e_{3}
\end{array}\right)
$$

in which $\varkappa_{1}$ is the initial twist, and $\varkappa_{2}$ and $\varkappa_{3}$ are the components of the initial curvature. In general, they are not constants, but functions of $\xi^{1}$.

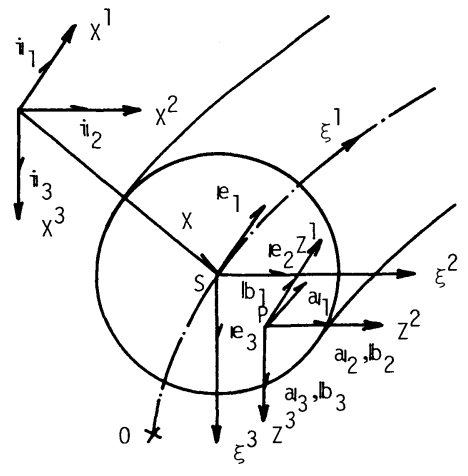

Fig. 1 Coordinate Systems.

The position vector at the point $\mathrm{P}$ is given by

$$
\boldsymbol{r}=\boldsymbol{x}+\xi^{2} \boldsymbol{e}_{2}+\xi^{3} \boldsymbol{e}_{3}
$$

The base vectors $\boldsymbol{a}_{m}$ at an arbitrary material point are obtained by differentiating the position vector $\boldsymbol{r}$ with respect to $\xi^{m}$,

$$
\boldsymbol{a}_{1}=\sqrt{g} \boldsymbol{e}_{1}-\varkappa_{1} \xi^{3} \boldsymbol{e}_{2}+\varkappa_{1} \xi^{2} \boldsymbol{e}_{3}, \quad \boldsymbol{a}_{2}=\boldsymbol{e}_{2}, \boldsymbol{a}_{3}=\boldsymbol{e}_{3}
$$

where

$$
\sqrt{g}=1+\varkappa_{2} \xi^{3}-\varkappa_{3} \xi^{2} \text {. }
$$

\section{FINITE ROTATION VECTORS}

According to Pietraszkiewicz and Badur ${ }^{20)}$, there exist the following three finite rotation vectors used in the existing literature :

$$
\omega=\omega \phi, \quad \Omega=\sin \omega \phi, \quad \theta=2 \tan \frac{\omega}{2} \phi
$$

where $\phi$ denotes the unit vector, the direction of which coincides with the direction of axis of the rotation, and $\omega$ the angle of rotation about its axis. Maeda and Hayashi ${ }^{5)}$ have used the finite rotation tensor to derive the finite displacement field in terms of six parameters. The finite rotation vector $\omega$ is quite equivalent to the finite rotation tensor. The finite rotation vector $\Omega$ is widely used in shell theory. On the other hand, there exist few papers in which the finite rotation vector $\theta$ is employed as a measure for finite rotations. The advantageous usage of the finite rotation vector $\theta$ has not been fully discussed. In this paper, the effects of finite rotations on the nonlinear behavior of rods are investigated with the help of the finite rotation vector $\theta$.

From Eq. (7.c) an applicable region of $\omega$ is defined by

$$
-\pi<\omega<\pi \text {. }
$$

Since, according to the assumption (3), the tangent unit base vectors after the deformation, $\overline{\boldsymbol{e}}_{m}$, remain orthogonal, we have the following relationships between $\boldsymbol{e}_{m}$ and $\overline{\boldsymbol{e}}_{m}{ }^{18.20)}$.

$$
\overline{\boldsymbol{e}}_{i}=\boldsymbol{e}_{i}+\frac{1}{2}(1+\cos \omega) \boldsymbol{\theta} \times \boldsymbol{e}_{i}+\frac{1}{4}(1+\cos \omega) \boldsymbol{\theta} \times\left(\boldsymbol{\theta} \times \boldsymbol{e}_{i}\right) .
$$

By expressing the vector $\theta$ into the component form with respect to the undeformed base vectors

$$
\boldsymbol{\theta}=\theta^{i} \boldsymbol{e}_{i},
$$

we can write the vectors $\overline{\boldsymbol{e}}_{m}$ in the form as

$$
\begin{aligned}
& \overline{\boldsymbol{e}}_{1}=\left[1-\frac{1}{4}(1+\cos \omega)\left\{\left(\theta^{2}\right)^{2}+\left(\theta^{3}\right)^{2}\right\}\right] \boldsymbol{e}_{1}+\frac{1}{2}(1+\cos \omega)\left(\theta^{3}+\frac{1}{2} \theta^{1} \theta^{2}\right) \boldsymbol{e}_{2} \\
& +\frac{1}{2}(1+\cos \omega)\left(-\theta^{2}+\frac{1}{2} \theta^{1} \theta^{3}\right) \boldsymbol{e}_{3}, \\
& \overline{\boldsymbol{e}}_{2}=\frac{1}{2}(1+\cos \omega)\left(-\theta^{3}+\frac{1}{2} \theta^{2} \theta^{1}\right) \boldsymbol{e}_{1}+\left[1-\frac{1}{4}(1+\cos \omega)\left\{\left(\theta^{3}\right)^{2}+\left(\theta^{1}\right)^{2}\right\}\right] \boldsymbol{e}_{2}
\end{aligned}
$$




$$
\begin{aligned}
& +\frac{1}{2}(1+\cos \omega)\left(\theta^{1}+\frac{1}{2} \theta^{2} \theta^{3}\right) \boldsymbol{e}_{3}, \\
& \overline{\boldsymbol{e}}_{3}=\frac{1}{2}(1+\cos \omega)\left(\theta^{2}+\frac{1}{2} \theta^{3} \theta^{1}\right) \boldsymbol{e}_{1}+\frac{1}{2}(1+\cos \omega)\left(-\theta^{1}+\frac{1}{2} \theta^{3} \theta^{2}\right) \boldsymbol{e}_{2} \\
& +\left[1-\frac{1}{4}(1+\cos \omega)\left\{\left(\theta^{1}\right)^{2}+\left(\theta^{2}\right)^{2}\right\}\right] \boldsymbol{e}_{3} .
\end{aligned}
$$

In view of Eqs. (7.c) and (10), we have

$$
\left(\theta^{1}\right)^{2}+\left(\theta^{2}\right)^{2}+\left(\theta^{3}\right)^{2}=\frac{4 \sin ^{2} \omega}{(1+\cos \omega)^{2}} .
$$

According to the assumption ( 3 ), the displacement vector at an arbitrary material point is presented by

$$
\boldsymbol{U}=\boldsymbol{u}+\xi^{2}\left(\overline{\boldsymbol{e}}_{2}-\boldsymbol{e}_{2}\right)+\xi^{3}\left(\overline{\boldsymbol{e}}_{3}-\boldsymbol{e}_{3}\right)
$$

where $\boldsymbol{u}$ denotes the displacement vector at the beam axis. By expressing the displacement vectors into the component form as

$$
\boldsymbol{U}=U^{i} \boldsymbol{e}_{i}, \quad \boldsymbol{u}=u^{i} \boldsymbol{e}_{i},
$$

we have the following displacement functions in terms of six parameters :

$$
\begin{aligned}
& U^{1}=u^{1}+\frac{1}{2} \xi^{2}(1+\cos \omega)\left(-\theta^{3}+\frac{1}{2} \theta^{2} \theta^{1}\right)+\frac{1}{2} \xi^{3}(1+\cos \omega)\left(\theta^{2}+\frac{1}{2} \theta^{3} \theta^{i}\right), \\
& U^{2}=u^{2}-\frac{1}{4} \xi^{2}(1+\cos \omega)\left[\left(\theta^{3}\right)^{2}+\left(\theta^{1}\right)^{2}\right]+\frac{1}{2} \xi^{3}(1+\cos \omega)\left(-\theta^{1}+\frac{1}{2} \theta^{3} \theta^{2}\right), \\
& U^{3}=u^{3}+\frac{1}{2} \xi^{2}(1+\cos \omega)\left(\theta^{1}+\frac{1}{2} \theta^{2} \theta^{3}\right)-\frac{1}{4} \xi^{3}(1+\cos \omega)\left[\left(\theta^{1}\right)^{2}+\left(\theta^{2}\right)^{2}\right] .
\end{aligned}
$$

\section{GEOMETRY AFTER DEFORMATION}

Since the tangent base vector to the beam axis after the deformation is given by differentiating the deformed position vector with respect to $\xi^{1}$, the tangent unit base vector $\overline{\boldsymbol{e}}_{1}$ takes the form as

$$
\overline{\boldsymbol{e}}_{1}=\frac{(\boldsymbol{x}+\boldsymbol{u})_{, 1}}{\left|(\boldsymbol{x}+\boldsymbol{u})_{, 1}\right|} \text {. }
$$

From Eq. (14. b), the vector $\overline{\boldsymbol{e}}_{1}$ can be written into the component form as

$$
\overline{\boldsymbol{e}}_{1}=\frac{1}{\sqrt{G_{0}}}\left\{\left(1+\left.u^{1}\right|_{1}\right) \boldsymbol{e}_{1}+\left.u^{2}\right|_{1} \boldsymbol{e}_{2}+\left.u^{3}\right|_{1} \boldsymbol{e}_{3}\right\}
$$

where $\left.()\right|_{1}$ denotes the covariant differentiation and

$$
\begin{array}{ll}
\left.u^{1}\right|_{1}=u_{, 1}^{1}-\varkappa_{3} u^{2}+\varkappa_{2} u^{3}, & \left.u^{2}\right|_{1}=u_{, 1}^{2}+\varkappa_{3} u^{1}-\varkappa_{1} u^{3}, \ldots \ldots \ldots . . . \\
\left.u^{3}\right|_{1}=u_{, 1}^{3}-\varkappa_{2} u^{1}+\varkappa_{1} u^{2}, & G_{0}=\left(1+\left.u^{1}\right|_{1}\right)^{2}+\left(\left.u^{2}\right|_{1}\right)^{2}+\left(\left.u^{3}\right|_{1}\right)^{2} .
\end{array}
$$

Comparison of the components of the base vector $\overline{\boldsymbol{e}}_{1}$ between Eqs. (11.a) and (17) leads to

$$
\begin{aligned}
& 1-\frac{1}{4}(1+\cos \omega)\left[\left(\theta^{2}\right)^{2}+\left(\theta^{3}\right)^{2}\right]=u^{1}\left\|_{1}, \frac{1}{2}(1+\cos \omega)\left(\theta^{3}+\frac{1}{2} \theta^{1} \theta^{2}\right)=u^{2}\right\|_{1}, \\
& \frac{1}{2}(1+\cos \omega)\left(-\theta^{2}+\frac{1}{2} \theta^{1} \theta^{3}\right)=u^{3} \|_{1}
\end{aligned}
$$

where

$$
u^{1}\left\|_{1}=\frac{1}{\sqrt{G_{0}}}\left(1+\left.u^{1}\right|_{1}\right), \quad u^{2}\right\|_{1}=\left.\frac{1}{\sqrt{G_{0}}} u^{2}\right|_{1}, \quad u^{3} \|_{1}=\left.\frac{1}{\sqrt{G_{0}}} u^{3}\right|_{1} .
$$

In Eqs. (19) there are three expressions for three unknowns $\theta^{i}$. However, with the help of Eqs. (12) and (18. d), it is shown that there exist only two independent expressions in Eqs. (19). Therefore, in addition to the displacement parameters $u^{i}$, we must choose a fourth parameter among the components of the finite rotation vector. Then we can present the remaining components of the finite rotation vector in terms of four parameters. In a linear theory, an angle of rotation of cross sections is chosen as a fourth parameter. In a nonlinear theory, on the other hand, there are various definitions for the fourth parameter. In would seem natural, in this paper, to introduce the following new variable $\vartheta$ as the fourth parameter associated with 
the rotation of cross sections:

$$
\theta^{1}=2 \tan \frac{1}{2} \vartheta \quad(-\pi<\vartheta<\pi) .
$$

It should be stressed that the applicable range of the present rotation parameter is twice that of Refs. ( 6 ) and (15). In the linear theory, the present fourth parameter $\vartheta$ coincides with an angle of rotation of cross sections.

From Eqs. (12), (19. a) and (21), it follows that

$$
\cos \omega=-1+\frac{1}{2}(1+\cos \vartheta)\left(1+u^{1} \|_{1}\right) \text {. }
$$

Introducing Eq. (22) into Eqs. (19. b) and (19. c), and using Eq. (12) leads to

$$
\theta^{2}=\lambda\left(u^{2}\left\|_{1} \frac{\sin \vartheta}{1+\cos \vartheta}-u^{3}\right\|_{1}\right), \quad \theta^{3}=\lambda\left(u^{2}\left\|_{1}+u^{3}\right\|_{1} \frac{\sin \vartheta}{1+\cos \vartheta}\right) \text {. }
$$

where

$$
\lambda=\frac{2}{1+u^{1} \|_{1}} .
$$

When Eqs. (21), (22) and (23) are substituted into Eqs. (15), the displacement functions become

$$
\begin{aligned}
& U^{1}=u^{1}-\xi^{2}\left(u^{2}\left\|_{1} \cos \vartheta+u^{3}\right\|_{1} \sin \vartheta\right)+\xi^{3}\left(u^{2}\left\|_{1} \sin \vartheta-u^{3}\right\|_{1} \cos \vartheta\right), \\
& U^{2}=u^{2}-\xi^{2}\left[\frac{1}{\lambda}(1-\cos \vartheta)+\lambda\left\{\frac{1}{4}\left(u^{2} \|_{1}\right)^{2}(1+\cos \vartheta)+\frac{1}{2} u^{2}\left\|_{1} u^{3}\right\|_{1} \sin \vartheta+\frac{1}{4}\left(u^{3} \|_{1}\right)^{2}(1-\cos \vartheta)\right\}\right] \\
& +\xi^{3}\left[-\frac{1}{\lambda} \sin \vartheta+\lambda\left\{\frac{1}{4}\left(u^{2} \|_{1}\right)^{2} \sin \vartheta-\frac{1}{2} u^{2}\left\|_{1} u^{3}\right\|_{1} \cos \vartheta-\frac{1}{4}\left(u^{3} \|_{1}\right)^{2} \sin \vartheta\right\}\right], \\
& U^{3}=u^{3}+\xi^{2}\left[\frac{1}{\lambda} \sin \vartheta+\lambda\left\{\frac{1}{4}\left(u^{2} \|_{1}\right)^{2} \sin \vartheta-\frac{1}{2} u^{2}\left\|_{1} u^{3}\right\|_{1} \cos \vartheta-\frac{1}{4}\left(u^{3} \|_{1}\right)^{2} \sin \vartheta\right\}\right]-\xi^{3}\left[\frac{1}{\lambda}(1-\cos \vartheta)\right. \\
& \left.+\lambda\left\{\frac{1}{4}\left(u^{2} \|_{1}\right)^{2}(1-\cos \vartheta)-\frac{1}{2} u^{2}\left\|_{1} u^{3}\right\|_{1} \sin \vartheta+\frac{1}{4}\left(u^{3} \|_{1}\right)^{2}(1+\cos \vartheta)\right\}\right] .
\end{aligned}
$$

The displacement function similar to Eq. (25. a) has been obtained in the previous papers. However it should be noted that the present displacement functions are derived without using any approximation. While the existing results are obtained by neglecting the higher order terms in the displacement functions. This difference may be caused by the choice of the fourth parameter. The displacement functions obtained herein do not violate the assumption that cross sections do not distort.

In accordance with Eq. (3), we introduce the notations defined by $\bar{\varkappa}_{1}=\overline{\boldsymbol{e}}_{2,1} \cdot \overline{\boldsymbol{e}}_{3}, \bar{\chi}_{2}=\overline{\boldsymbol{e}}_{3,1} \cdot \overline{\boldsymbol{e}}_{1}, \bar{\varkappa}_{3}=\overline{\boldsymbol{e}}_{1,1} \cdot \overline{\boldsymbol{e}}_{2}$

These notations are not exactly the twist and curvatures since the deformed line is extended. However, under the small strain assumptions, $\bar{\chi}_{1}$ denotes the twist, and $\bar{\chi}_{2}$ and $\bar{\chi}_{3}$ denote the components of the curvature after the deformation. Substituting Eqs. (11) into Eqs. (26) leads to

$$
\begin{aligned}
& \bar{\varkappa}_{1}=\frac{1}{2}(1+\cos \omega) \theta_{, 1}^{1}+\frac{1}{4}(1+\cos \omega) \theta^{3} \theta_{, 1}^{2}-\frac{1}{4}(1+\cos \omega) \theta^{2} \theta_{,{ }_{1}}, \\
& \bar{\varkappa}_{2}=\frac{1}{2}(1+\cos \omega) \theta_{, 1}^{2}+\frac{1}{4}(1+\cos \omega) \theta^{1} \theta_{, 1}^{3}-\frac{1}{4}(1+\cos \omega) \theta^{3} \theta_{,{ }_{1},}, \\
& \bar{\varkappa}_{3}=\frac{1}{2}(1+\cos \omega) \theta_{, 1}^{3}+\frac{1}{4}(1+\cos \omega) \theta^{2} \theta_{, 1}^{1}-\frac{1}{4}(1+\cos \omega) \theta^{1} \theta_{,{ }_{1}}{ }^{2},
\end{aligned}
$$

When Eqs. (21) to (23) are introduced into Eqs. (27), $\bar{\chi}_{i}$ are expressed as

$$
\begin{aligned}
& \bar{\varkappa}_{1}=\left[\frac{1}{\lambda}+\frac{1}{4} \lambda\left\{\left(u^{2} \|_{1}\right)^{2}+\left(u^{3} \|_{1}\right)^{2}\right\}\right] \vartheta_{, 1}+\frac{1}{2} \lambda\left[\left(u^{2} \|_{1}\right)_{, 1} u^{3}\left\|_{1}-\left(u^{3} \|_{1}\right)_{, 1} u^{2}\right\|_{1}\right]+\varkappa_{1} u^{1}\left\|_{1}+\varkappa_{2} u^{2}\right\|_{1}+\varkappa_{3} u^{3} \|_{1} \text {, } \\
& \bar{\chi}_{2}=-\frac{1}{2} \lambda\left(u^{2}\left\|_{1} \sin \vartheta-u^{3}\right\|_{1} \cos \vartheta\right)\left(u^{1} \|_{1}\right)_{, 1}+\left(u^{2} \|_{1}\right)_{, 1} \sin \vartheta-\left(u^{3} \|_{1}\right)_{1,} \cos \vartheta-\varkappa_{1}\left(u^{2}\left\|_{1} \cos \vartheta+u^{3}\right\|_{1} \sin \vartheta\right) \\
& +\varkappa_{2}\left[1+\frac{1}{\lambda}(\cos \vartheta-1)-\lambda\left\{\frac{1}{4}\left(u^{2} \|_{1}\right)^{2}(1+\cos \vartheta)+\frac{1}{2} u^{2}\left\|_{1} u^{3}\right\|_{1} \sin \vartheta+\frac{1}{4}\left(u^{3} \|_{1}\right)^{2}(1-\cos \vartheta)\right\}\right] \\
& +\varkappa_{3}\left[\frac{1}{\lambda} \sin \vartheta+\lambda\left\{\frac{1}{4}\left(u^{2} \|_{1}\right)^{2} \sin \vartheta-\frac{1}{2} u^{2}\left\|_{1} u^{3}\right\|_{1} \cos \vartheta-\frac{1}{4}\left(u^{3} \|_{1}\right)^{2} \sin \vartheta\right\}\right],
\end{aligned}
$$




$$
\begin{aligned}
& \bar{\varkappa}_{3}=-\frac{1}{2} \lambda\left(u^{2}\left\|_{1} \cos \vartheta+u^{3}\right\|_{1} \sin \vartheta\right)\left(u^{1} \|_{1}\right)_{1}+\left(u^{2} \|_{1}\right)_{1} \cos \vartheta+\left(u^{3} \|_{1}\right)_{1} \sin \vartheta+\varkappa_{1}\left(u^{2}\left\|_{1} \sin \vartheta-u^{3}\right\|_{1} \cos \vartheta\right) \\
& +\varkappa_{2}\left[-\frac{1}{\lambda} \sin \vartheta+\lambda\left\{\frac{1}{4}\left(u^{2} \|_{1}\right)^{2} \sin \vartheta-\frac{1}{2} u^{2}\left\|_{1} u^{3}\right\|_{1} \cos \vartheta-\frac{1}{4}\left(u^{3} \|_{1}\right)^{2} \sin \vartheta\right\}\right]+\varkappa_{3}\left[1-\frac{1}{\lambda}(1-\cos \vartheta)\right. \\
& \left.-\lambda\left\{\frac{1}{4}\left(u^{2} \|_{1}\right)^{2}(1-\cos \vartheta)-\frac{1}{2} u^{2}\left\|_{1} u^{3}\right\|_{1} \sin \vartheta+\frac{1}{4}\left(u^{3} \|_{1}\right)^{2}(1+\cos \vartheta)\right\}\right] .
\end{aligned}
$$

The above expressions are obtained without restricting the magnitude of displacements or angles of rotation. While, in Refs. (6) and (15), the fourth and higher order terms with respect to displacement components are neglected in calculating the twist and curvatures after the deformation.

\section{INTERNAL AND EXTERNAL VIRTUAL WORKS}

The Green strain tensors associated with the directions of the $\xi^{i}$ lines are given by

$$
e_{i j}=\frac{1}{2}\left(\overline{\boldsymbol{a}}_{i} \cdot \overline{\boldsymbol{a}}_{j}-\boldsymbol{a}_{i} \boldsymbol{a}_{j}\right)
$$

where

$$
\overline{\boldsymbol{a}}_{1}=\left(\sqrt{G_{0}}-\bar{\varkappa}_{3} \xi^{2}+\bar{\varkappa}_{2} \xi^{3}\right) \overline{\boldsymbol{e}}_{1}-\bar{\varkappa}_{1} \xi^{3} \overline{\boldsymbol{e}}_{2}+\bar{\varkappa}_{1} \xi^{2} \overline{\boldsymbol{e}}_{3}, \quad \overline{\boldsymbol{a}}_{2}=\overline{\boldsymbol{e}}_{2}, \quad \overline{\boldsymbol{a}}_{3}=\overline{\boldsymbol{e}}_{3 .}
$$

Substituting Eqs. (30) into Eq. (29) implies

$$
\begin{gathered}
e_{11}=\left(\varepsilon_{0}-\xi^{2} \varkappa_{3}^{*}+\xi^{3} \varkappa_{2}^{*}\right)\left\{\sqrt{g}+\frac{1}{2}\left(\varepsilon_{0}-\xi^{2} \varkappa_{3}^{*}+\xi^{3} \varkappa_{2}^{*}\right)\right\}+\frac{1}{2} \rho^{2} \chi_{1}^{*}\left(x_{1}^{*}+2 \varkappa_{1}\right), \quad e_{12}=e_{21}=-\frac{1}{2} x_{1}^{*} \xi^{3}, \\
e_{13}=e_{31}=\frac{1}{2} x_{1}^{*} \xi^{2}, \quad e_{22}=e_{23}=e_{32}=e_{33}=0 .
\end{gathered}
$$

where

$$
\varepsilon_{0}=\sqrt{G_{0}}-1, \quad \chi_{i}^{*}=\bar{\varkappa}_{i}-\varkappa_{i}, \quad \rho^{2}=\left(\xi^{2}\right)^{2}+\left(\xi^{3}\right)^{2} .
$$

Let $\tau^{i j}$ denote the second Piola-Kirchhoff stress tensors associated with the directions of the $\xi^{i}$ lines. Then the internal virtual work can be put in the form $a^{21)}$

$$
I V W=\int_{V}\left(\tau^{11} \delta e_{11}+2 \tau^{12} \delta e_{12}+2 \tau^{13} \delta e_{13}\right) d V
$$

where

$$
d V=\sqrt{g} d \xi^{1} d \xi^{2} d \xi^{3}
$$

Since the base vectors $\boldsymbol{a}_{i}$ are not orthogonal, the constitutive equations may become complicated. Therefore, for the latter convenience, we introduce the strain tensors $\varepsilon_{i j}$ and the stress tensors $\sigma^{i j}$ associated with the coordinates $Z^{i}$. The relationships between the strain tensors $e_{i j}$ and $\varepsilon_{i j}$ are given by

$$
\varepsilon_{i j}=\frac{\partial \xi^{m}}{\partial Z^{i}} \cdot \frac{\partial \xi^{n}}{\partial Z^{j}} e_{m n}
$$

where

$$
\frac{\partial \xi^{1}}{\partial Z^{1}}=\frac{1}{\sqrt{g}}, \frac{\partial \xi^{2}}{\partial Z^{1}}=\frac{\varkappa_{1} \xi^{3}}{\sqrt{g}}, \frac{\partial \xi^{3}}{\partial Z^{1}}=-\frac{\varkappa_{1} \xi^{2}}{\sqrt{g}}, \frac{\partial \xi^{2}}{\partial Z^{2}}=\frac{\partial \xi^{3}}{\partial Z^{3}}=1, \frac{\partial \xi^{1}}{\partial Z^{2}}=\frac{\partial \xi^{3}}{\partial Z^{2}}=\frac{\partial \xi^{1}}{\partial Z^{3}}=\frac{\partial \xi^{2}}{\partial Z^{3}}=0 .
$$

Substituting Eqs. (31) into (35), and using Eqs. (36) leads to

$$
\begin{aligned}
& \varepsilon_{11}=\left(\varepsilon_{0}-\xi^{2} \varkappa_{3}^{*}+\xi^{3} \varkappa_{2}^{*}\right)\left\{\frac{1}{\sqrt{g}}+\frac{1}{2 g}\left(\varepsilon_{0}-\xi^{2} \varkappa_{3}^{*}+\xi^{3} \varkappa_{2}^{*}\right)\right\}+\frac{1}{2 g} \rho^{2}\left(\varkappa_{1}^{*}\right)^{2}, \quad \varepsilon_{12}=\varepsilon_{21}=-\frac{\xi^{3} \varkappa_{1}^{*}}{2 \sqrt{g}}, \\
& \varepsilon_{13}=\varepsilon_{31}=\frac{\xi^{2} \varkappa_{1}^{*}}{2 \sqrt{g}}, \quad \varepsilon_{22}=\varepsilon_{23}=\varepsilon_{32}=\varepsilon_{33}=0 .
\end{aligned}
$$

The physical component of the stress tensors $\sigma^{i j}$ is given by ${ }^{15)}$

$$
\tilde{\sigma}^{i j}=\sigma^{i j} \sqrt{b_{i i}} \sqrt{b_{j j}} \quad(i, j \text { not summed })
$$

where 


$$
b_{i j}=\boldsymbol{b}_{i} \cdot \boldsymbol{b}_{j}, \quad \bar{b}_{i j}=\overline{\boldsymbol{b}}_{i} \cdot \overline{\boldsymbol{b}}_{j}, \quad \boldsymbol{b}_{i}=\boldsymbol{e}_{i}, \quad \overline{\boldsymbol{b}}_{1}=\frac{1}{\sqrt{g}}\left(\sqrt{g}+\varepsilon_{0}-\xi^{2} \varkappa_{3}^{*}+\xi^{3} \varkappa_{2}^{*}\right) \overline{\boldsymbol{e}}_{1}, \quad \overline{\boldsymbol{b}}_{2}=\overline{\boldsymbol{e}}_{2}, \overline{\boldsymbol{b}}_{3}=\overline{\boldsymbol{e}}_{3} . \quad \cdots(39 . \mathrm{a}-\mathrm{f})
$$

The relationships between the stress tensors $\tau^{i j}$ and $\sigma^{i j}$ are expressed by

$$
\sigma^{i j}=\frac{\partial Z^{i}}{\partial \xi^{m}} \frac{\partial Z^{j}}{\partial \xi^{n}} \tau^{m n}
$$

With the use of Eqs. (33), (35), (38) and (40), the internal virtual work can be rewritten in the form as

$$
I V W=\int_{V}\left[\frac{\tilde{\sigma}^{11}}{\sqrt{g}}\left\{\delta \varepsilon_{0}-\xi^{2} \delta \varkappa_{3}^{*}+\xi^{3} \delta \varkappa_{2}^{*}+\frac{\rho^{2} \varkappa_{1}^{*} \delta \varkappa_{1}^{*}}{\underline{\sqrt{g}+\varepsilon_{0}-\xi^{2} \varkappa_{3}^{*}+\xi^{3} \varkappa_{2}^{*}}}\right\}+\frac{1}{\sqrt{g}}\left(-\tilde{\sigma}^{12} \xi^{3}+\tilde{\sigma}^{13} \xi^{2}\right) \delta \varkappa_{1}^{*}\right] d V .
$$

When a rod is subject to the distributed force vector, $\boldsymbol{p}=p_{i} \boldsymbol{e}_{i}$, along the beam axis and the distributed surface force vector, $\overline{\boldsymbol{P}}=\bar{P}_{i} \boldsymbol{e}_{i}$, at the end cross sections, the external virtual work is given by

$$
E V W=\int_{V} P_{i} \delta U^{i} d V+\left[\int_{A} \bar{p}_{i} \delta U^{i} d A\right]_{\xi^{1}=\xi_{* *}{ }^{1}=\xi_{*}^{1}}^{\xi^{1}}
$$

where

$$
d A=d \xi^{2} d \xi^{3}
$$

and $\xi_{*}^{1}$ and $\xi_{* *}^{1}$ denote the coordinates of $\xi^{1}$ at both ends of the rod.

\section{SECOND ORDER THEORY OF ELASTIC RODS}

In the previous sections, we derive the exact finite displacement field of naturally curved and twisted rods, and the exact twist and curvatures after the deformation on the basis of the Bernoulli-Euler hypothesis. The exact internal and external virtual works are presented in terms of the physical components of stress tensors. When we derive these fundamental equations, we do not restrict the magnitude of displacements or angles of rotation, nor utilize the small-strain assumptions. However it is found difficult from Eq. (41) to define the appropriate stress resultants and moments, since not only the coordinates but also the extension and the curvatures after the deformation are included in the denominator of the underlined term in Eq. (41). As discussed in Ref. (6), it seems to be few problems in which the third and higher order terms with respect to displacement components must be taken into account in the governing equations. In view of these facts, we supplement the following assumptions to develop a geometrically nonlinear theory of elastic rods :

(4) Strains are small in comparison with unity.

(5) The third and higher order terms with respect to displacement components are neglected in the displacement functions, and the twist and curvatures after the deformation. However the rotation parameter $\vartheta$ remains to be finite. In the coefficients of the stress resultants and moments in the equilibrium equations and the associated boundary conditions, the second and higher order terms with respect to displacement parameters are neglected.

As pointed out in Refs. (6) and (7), the assumption (4) is employed after taking first variations with respect to displacement and rotation parameters so that the second order terms are fully taken into account in the governing equations.

According to the assumption (5), Eq. (24) yields the following approximation :

$$
\lambda=1+\frac{1}{4}\left[\left(u^{2} \|_{1}\right)^{2}+\left(u^{3} \|_{1}\right)^{2}\right] \text {. }
$$

In what follows, the notations $u^{i} \|_{1}$ are still used. However it should be noted that the third and higher order terms in $u^{i} \|_{1}$ are meaningless. Substituting Eq. (44) into Eq. (25), and using the assumption (5) leads to

$$
\begin{aligned}
& U^{1}=u^{1}-\xi^{2}\left(u^{2}\left\|_{1} \cos \vartheta+u^{3}\right\|_{1} \sin \vartheta\right)+\xi^{3}\left(u^{2}\left\|_{1} \sin \vartheta-u^{3}\right\|_{1} \cos \vartheta\right), \\
& U^{2}=u^{2}-\xi^{2}\left\{1-\cos \vartheta+\frac{1}{2} u^{2}\left\|_{1} u^{3}\right\|_{1} \sin \vartheta+\frac{1}{2}\left(u^{2} \|_{1}\right)^{2} \cos \vartheta\right\}-\xi^{3}\left\{\sin \vartheta+\frac{1}{2} u^{2}\left\|_{1} u^{3}\right\|_{1} \cos \vartheta\right.
\end{aligned}
$$


$\left.-\frac{1}{2}\left(u^{2} \|_{1}\right)^{2} \sin \vartheta\right\}$

$U^{3}=u^{3}+\xi^{2}\left\{\sin \vartheta-\frac{1}{2} u^{2}\left\|_{1} u^{3}\right\|_{1} \cos \vartheta-\frac{1}{2}\left(u^{3} \|_{1}\right)^{2} \sin \vartheta\right\}-\xi^{3}\left\{1-\cos \vartheta-\frac{1}{2} u^{2}\left\|_{1} u^{3}\right\|_{1} \sin \vartheta\right.$

$+\frac{1}{2}\left(u^{3} \|_{1}\right)^{2} \cos \vartheta$.

Introducing Eq. (44) into Eq. (28), and using the assumption ( 5 ) leads to

$$
\begin{aligned}
& \bar{\varkappa}_{1}=\vartheta_{, 1}+\frac{1}{2}\left\{\left(u^{2} \|_{1}\right)_{1} u^{3}\left\|_{1}-\left(u^{3} \|_{1}\right)_{1} u^{2}\right\|_{1}\right\}+\varkappa_{1} u^{1}\left\|_{1}+\varkappa_{2} u^{2}\right\|_{1}+\varkappa_{3} u^{3} \|_{1} \text {, } \\
& \bar{\varkappa}_{2}=\left(u^{2} \|_{1}\right)_{1} \sin \theta-\left(u^{3} \|_{1}\right)_{1} \cos \theta-\varkappa_{1}\left(u^{2}\left\|_{1} \cos \theta+u^{3}\right\|_{1} \sin \theta\right)+\varkappa_{2}\left\{\cos \theta-\frac{1}{2} u^{2}\left\|_{1} u^{3}\right\|_{1} \sin \theta\right.
\end{aligned}
$$$$
\left.-\frac{1}{2}\left(u^{2} \|_{1}\right)^{2} \cos \vartheta\right\}+\varkappa_{3}\left[\sin \vartheta-\frac{1}{2} u^{2}\left\|_{1} u^{3}\right\|_{1} \cos \vartheta-\frac{1}{2}\left(u^{3} \|_{1}\right)^{2} \sin \vartheta\right],
$$

$\bar{\varkappa}_{3}=\left(u^{2} \|_{1}\right)_{1} \cos \vartheta+\left(u^{3} \|_{1}\right)_{1} \sin \vartheta+\varkappa_{1}\left(u^{2}\left\|_{1} \sin \vartheta-u^{3}\right\|_{1} \cos \vartheta\right)+\varkappa_{2}\left\{-\sin \vartheta-\frac{1}{2} u^{2}\left\|_{1} u^{3}\right\|_{1} \cos \vartheta\right.$

$\left.+\frac{1}{2}\left(u^{2} \|_{1}\right)^{2} \sin \vartheta\right\}+\varkappa_{3}\left[\cos \vartheta+\frac{1}{2} u^{2}\left\|_{1} u^{3}\right\|_{1} \sin \vartheta-\frac{1}{2}\left(u^{3} \|_{1}\right)^{2} \cos \vartheta\right]$.

The internal virtual work, according to the assumption (4), takes the form as

$$
I V W=\int_{V}\left[\frac{\tilde{\sigma}^{11}}{\sqrt{g}}\left\{\delta \varepsilon_{0}-\xi^{2} \delta \varkappa_{3}^{*}+\xi^{3} \delta \varkappa_{2}^{*}+\frac{\rho^{2}}{\sqrt{g}} \varkappa_{1}^{*} \delta \varkappa_{1}^{*}\right\}+\frac{1}{\sqrt{g}}\left(-\tilde{\sigma}^{12} \xi^{3}+\tilde{\sigma}^{13} \xi^{2}\right) \delta \varkappa_{1}^{*}\right] d V .
$$

The external virtual work is evaluated by using the displacement funcuions expressed by Eqs. (45) .

The equilibrium equations and the associated boundary conditions are derived from the principle of virtual work which asserts that $I V W=E V W$. For the sake of convenience, the second and higher order terms with respect to displacement components are included implicitly in the coefficients of stress resultants and moments. These higher order terms, however, are meaningless since the assumption (5) are introduced to obtain the governing equations.

The equilibrium equations are represented by

$$
\begin{aligned}
& \left(N-Q_{2} u^{2}\left\|_{1}-Q_{3} u^{3}\right\|_{1}\right)_{1}+\varkappa_{2}\left(N u^{3} \|_{1}+Q_{3}\right)-\varkappa_{3}\left(N u^{2} \|_{1}+Q_{2}\right)+N^{*}+M_{\xi, 1}^{*}+\varkappa_{2} M_{\varphi}^{*}+\varkappa_{3} M_{\psi}^{*}=0 \quad\left(\delta u^{1}\right), \\
& \left(N u^{2} \|_{1}+Q_{2}\right)_{1}-\varkappa_{1}\left(N u^{3} \|_{1}+Q_{3}\right)+\varkappa_{3}\left(N-Q_{2} u^{2}\left\|_{1}-Q_{3} u^{3}\right\|_{1}\right)+Q_{2}^{*}-M_{\psi, 1}^{*}-\varkappa_{1} M_{\varphi}^{*}+\varkappa_{3} M_{5}^{*}=0 \quad\left(\delta u^{2}\right), \\
& \left.Q_{2} u^{2}\left\|_{1}-Q_{3} u^{3}\right\|_{1}\right)+Q_{3}^{*}+M_{\varphi, 1}^{*}-\chi_{1} M_{\psi}^{*}-\varkappa_{2} M_{\xi}^{*}=0 \quad\left(\delta u^{3}\right) \\
& \left(N u^{3} \|_{1}+Q_{3}\right)_{, 1}+\varkappa_{1}\left(N u^{2} \|_{1}+Q_{2}\right)-\varkappa_{2}\left(N-Q_{2} u^{2}\left\|_{1}-Q_{3} u^{3}\right\|_{1}\right)+Q_{3}^{*}+M_{\varphi, 1}^{*}-\varkappa_{1} M_{\psi}^{*}-\varkappa_{2} M_{\xi}^{*}=0 \quad\left(\delta u^{3}\right), \\
& T_{, 1}+\bar{\varkappa}_{2} M_{3}-\bar{\varkappa}_{3} M_{2}+T^{*}+M_{n}^{*}=0 \quad(\delta \vartheta) \\
& N=\int \tilde{\sigma}^{11} d A, \quad M_{2}=\int \tilde{\sigma}^{11} \xi^{3} d A, \quad M_{3}=-\int \tilde{\sigma}^{11} \xi^{2} d A, \quad M_{t}=\int\left(-\tilde{\sigma}^{12} \xi^{3}+\tilde{\sigma}^{13} \xi^{2}\right) d A, \\
& M_{\rho}=\int \frac{1}{\sqrt{g}} \tilde{\sigma}^{11} \rho^{2} d A, \quad T=M_{t}+M_{\rho} x_{1}^{*}, \quad M_{x}=M_{2} \cos \vartheta-M_{3} \sin \vartheta, \quad M_{y}=M_{2} \sin \vartheta+M_{3} \cos \vartheta, \\
& Q_{2}=-M_{y, 1}-T\left(u^{3} \|_{1}\right)_{1}-\varkappa_{1}\left(M_{x}+T u^{2} \|_{1}\right)+\varkappa_{2}\left(T-M_{x} u^{2} \|_{1}\right)-\varkappa_{3} M_{x} u^{3}\left\|_{1}-\frac{1}{2}\left(T_{, 1}+\varkappa_{2} M_{y}-\varkappa_{3} M_{x}\right) u^{3}\right\|_{1}, \\
& Q_{3}=M_{x, 1}+T\left(u^{2} \|_{1}\right)_{1}-\varkappa_{1}\left(M_{y}+T u^{3} \|_{1}\right)-\varkappa_{2} M_{y} u^{2}\left\|_{1}+\varkappa_{3}\left(T-M_{y} u^{3} \|_{1}\right)+\frac{1}{2}\left(T_{, 1}+\varkappa_{2} M_{y}-\varkappa_{3} M_{x}\right) u^{2}\right\|_{1}
\end{aligned}
$$

and

$N^{*}=\int P_{1} \sqrt{g} d A, \quad M_{2}^{*}=\int P_{1} \xi^{3} \sqrt{g} d A, \quad M_{3}^{*}=-\int P_{1} \xi^{2} \sqrt{g} d A, \quad Q_{2}^{*}=\int P_{2} \sqrt{g} d A, \quad Q_{3}^{*}=\int P_{3} \sqrt{g} d A$, $T_{22}^{*}=-\int P_{2} \xi^{2} \sqrt{g} d A, \quad T_{23}^{*}=-\int P_{2} \xi^{3} \sqrt{g} d A, \quad T_{32}^{*}=\int P_{3} \xi^{2} \sqrt{g} d A, \quad T_{33}^{*}=-\int P_{3} \xi^{3} \sqrt{g} d A$, $T_{x x}^{*}=T_{22}^{*} \sin \vartheta+T_{23}^{*} \cos \vartheta, \quad T_{x y}^{*}=T_{22}^{*} \cos \vartheta-T_{23}^{*} \sin \vartheta, \quad T_{y x}^{*}=T_{33}^{*} \cos \vartheta-T_{32}^{*} \sin \vartheta$, 


$$
\begin{aligned}
& T_{y y}^{*}=T_{32}^{*} \cos \vartheta+T_{33}^{*} \sin \vartheta, \quad T^{*}=T_{x x}^{*}+T_{y y}^{*}, \quad M_{x}^{*}=M_{2}^{*} \cos \vartheta-M_{3}^{*} \sin \vartheta, \\
& M_{y}^{*}=M_{2}^{*} \sin \vartheta+M_{3}^{*} \cos \vartheta, \quad M_{\xi}^{*}=-M_{x}^{*} u^{3}\left\|_{1}+M_{y}^{*} u^{2}\right\|_{1}, \quad M_{n}^{*}=M_{x}^{*} u^{2}\left\|_{1}+M_{y}^{*} u^{3}\right\|_{1}, \\
& M_{\varphi}^{*}=M_{x}^{*}-\frac{1}{2} T_{x x}^{*} u^{2}\left\|_{1}+\frac{1}{2} T_{y y}^{*} u^{2}\right\|_{1}-T_{y x}^{*} u^{3}\left\|_{1}, \quad M_{\psi}^{*}=M_{y}^{*}+\frac{1}{2} T_{x x}^{*} u^{3}\right\|_{1}-\frac{1}{2} T_{y}^{*} u^{3}\left\|_{1}+T_{x y}^{*} u^{2}\right\|_{1} .
\end{aligned}
$$

The boundary conditions at both end cross sections are given by

$$
\begin{aligned}
& N-Q_{2} u^{2}\left\|_{1}-Q_{3} u^{3}\right\|_{1}=\bar{N}^{*}-M_{\xi}^{*} \text { or } u^{1}=\bar{u}^{1} \text {, } \\
& N u^{2} \|_{1}+Q_{2}=\bar{Q}_{2}^{*}+M_{\psi}^{*} \text { or } u^{2}=\bar{u}^{2} \text {, } \\
& N u^{3} \|_{1}+Q_{3}=\bar{Q}_{3}^{*}-M_{\varphi}^{*} \text { or } u^{3}=\bar{u}^{3} \text {, } \\
& T=\bar{T}^{*}+\bar{M}_{n}^{*} \text { or } \vartheta=\bar{\vartheta} \text {, } \\
& M_{y}+\frac{1}{2} T u^{3} \|_{1}=\bar{M}_{\psi}^{*} \text { or } u^{2}\left\|_{1}=\bar{u}^{2}\right\|_{1} \text {, } \\
& M_{x}+\frac{1}{2} T u^{2} \|_{1}=\bar{M}_{\varphi}^{*} \text { or } u^{3}\left\|_{1}=\bar{u}^{3}\right\|_{1} \text {. }
\end{aligned}
$$

where $\bar{u}^{i}, \bar{\vartheta}, \bar{u}^{2} \|_{1}$ and $\bar{u}^{3} \|_{1}$ are prescribed values at both end cross sections and

$$
\begin{aligned}
& \bar{N}^{*}=\int \bar{P}_{1} d A, \bar{M}_{2}^{*}=\int \bar{P}_{1} \xi^{3} d A, \bar{M}_{3}^{*}=-\int \bar{P}_{1} \xi^{2} d A, \bar{Q}_{2}^{*}=\int \bar{P}_{2} d A, \bar{Q}_{3}^{*}=\int \bar{P}_{3} d A, \bar{T}_{22}^{*}=-\int \bar{P}_{2} \xi^{2} d A, \\
& \bar{T}_{23}^{*}=-\int \bar{P}_{2} \xi^{3} d A, \bar{T}_{32}^{*}=\int \bar{P}_{3} \xi^{2} d A, \bar{T}_{33}^{*}=-\int \bar{P}_{3} \xi^{3} d A, \bar{T}_{x x}^{*}=\bar{T}_{22}^{*} \sin \vartheta+\bar{T}_{23}^{*} \cos \vartheta, \\
& \bar{T}_{x y}^{*}=\bar{T}_{22}^{*} \cos \vartheta-\bar{T}_{23}^{*} \sin \vartheta, \bar{T}_{y x}^{*}=\bar{T}_{33}^{*} \cos \vartheta-\bar{T}_{32}^{*} \sin \vartheta, \bar{T}_{y y}^{*}=\bar{T}_{32}^{*} \cos \vartheta+\bar{T}_{33}^{*} \sin \vartheta, \\
& \bar{T}^{*}=\bar{T}_{x x}^{*}+\bar{T}_{y y}^{*}, \bar{M}_{x}^{*}=\bar{M}_{2}^{*} \cos \vartheta-\bar{M}_{3}^{*} \sin \vartheta, \bar{M}_{y}^{*}=\bar{M}_{2}^{*} \sin \vartheta+\bar{M}_{3}^{*} \cos \vartheta, \\
& \bar{M}_{n}^{*}=\bar{M}_{x}^{*} u^{2}\left\|_{1}+\bar{M}_{y}^{*} u^{3}\right\|_{1}, \bar{M}_{\varphi}^{*}=\bar{M}_{x}^{*}-\frac{1}{2} \bar{T}_{x x}^{*} u^{2}\left\|_{1}+\frac{1}{2} \bar{T}_{y y}^{*} u^{2}\right\|_{1}-\bar{T}_{y x}^{*} u^{3} \|_{1}, \\
& \bar{M}_{\psi}^{*}=\bar{M}_{y}^{*}+\frac{1}{2} \bar{T}_{x x}^{*} u^{3}\left\|_{1}-\frac{1}{2} \bar{T}_{y y}^{*} u^{3}\right\|_{1}+\bar{T}_{x y}^{*} u^{2} \|_{1} .
\end{aligned}
$$

Since the strains are assumed to be small in comparison with unity, the stress-strain relationships for elastic rods are written as

$$
\tilde{\sigma}^{11}=E \tilde{\varepsilon}_{11}, \quad \tilde{\sigma}^{12}=2 G \tilde{\varepsilon}_{12}, \quad \tilde{\sigma}^{13}=2 G \tilde{\varepsilon}_{13}
$$

where $E$ denotes the Young modulus, $G$ the shear modulus, $\tilde{\varepsilon}_{i j}$ the physical component of strain tensors. Introducing Eqs. (37) and (53) into Eqs. (49), and integrating over cross sections leads to

$$
\begin{aligned}
& N=E\left[F_{1} \varepsilon_{0}-F_{2} \varkappa_{3}^{*}+F_{3} \varkappa_{2}^{*}+\frac{1}{2} F_{\rho}\left(\varkappa_{1}^{*}\right)^{2}\right], \quad M_{2}=E\left[F_{3} \varepsilon_{0}-I_{23} \varkappa_{3}^{*}+I_{33} \varkappa_{2}^{*}+\frac{1}{2} F_{\rho 3}\left(\varkappa_{1}^{*}\right)^{2}\right], \\
& M_{3}=E\left[-F_{2} \varepsilon_{0}+I_{22} \varkappa_{3}^{*}-I_{23} \varkappa_{2}^{*}+\frac{1}{2} F_{\rho 2}\left(\varkappa_{1}^{*}\right)^{2}\right], \quad M_{\rho}=E\left[F_{\rho} \varepsilon_{0}-F_{\rho 2} \varkappa_{3}^{*}+F_{\rho 3} \varkappa_{2}^{*}+\frac{1}{2} F_{\rho \rho}\left(\varkappa_{1}^{*}\right)^{2}\right], \\
& M_{t}=G J \varkappa_{1}^{*}
\end{aligned}
$$

where

$$
\begin{aligned}
& F_{1}=\int \frac{1}{\sqrt{g}} d A, \quad F_{2}=\int \frac{\xi^{2}}{\sqrt{g}} d A, \quad F_{3}=\int \frac{\xi^{3}}{\sqrt{g}} d A, \quad F_{\rho}=\int \frac{\rho^{2}}{g} d A, \quad F_{\rho_{2}}=\int \frac{\rho^{2} \xi^{2}}{g} d A, \\
& F_{\rho 3}=\int \frac{\rho^{2} \xi^{3}}{g} d A, \quad F_{\rho \rho}=\int \frac{\rho^{4}}{g \sqrt{g}} d A, \quad I_{22}=\int \frac{1}{\sqrt{g}}\left(\xi^{2}\right)^{2} d A, \quad I_{23}=\int \frac{1}{\sqrt{g}} \xi^{2} \xi^{3} d A, \\
& I_{33}=\int \frac{1}{\sqrt{g}}\left(\xi^{3}\right)^{2} d A, \quad J=\int \frac{1}{\sqrt{g}} \rho^{2} d A .
\end{aligned}
$$

\section{DISCUSSION}

In order to confirm the validity of the present equations, comparison is made between the present equilibrium equations and another ones obtained from the equilibrium method. The assumptions (1) to 
(5) are employed and the distributed external forces are assumed to be zero for simplicity.

Let $N$ and $M$ denote the resultant force vector and moment vector, respectively, represented by $\boldsymbol{N}=N \overline{\boldsymbol{e}}_{1}+q_{2} \overline{\boldsymbol{e}}_{2}+q_{3} \overline{\boldsymbol{e}}_{3}, \quad \boldsymbol{M}=T \overline{\boldsymbol{e}}_{1}+M_{2} \overline{\boldsymbol{e}}_{2}+M_{3} \overline{\boldsymbol{e}}_{3}$

where $q_{2}$ and $q_{3}$ denote the shear forces in the directions of the deformed principal axes. It should be noted that the quantities $Q_{2}$ and $Q_{3}$ defined by Eqs. (49. i) and (49. j) are not the same as the shear forces $q_{2}$ and $q_{3}$. In this paper, the shear deformation due to bending are assumed to be neglected so that the shear forces, defined by integration of shear stresses over cross sections, become zero. As a result, in the case of using the energy method, the shear forces do not appear explicitly in the equilibrium equations. Therefore comparison of equilibrium equations is made in terms of the axial force $N$ and moments $T, M_{2}$, $M_{3}$. The relationships between the shear forces $q_{2}, q_{3}$ and the quantities $Q_{2}, Q_{3}$ will be discussed later.

The equilibrium conditions of a deformed rod element take the form as

$$
\frac{d \boldsymbol{N}}{d \xi^{1}}=0, \frac{d \boldsymbol{M}}{d \xi^{1}}+\overline{\boldsymbol{e}}_{1} \times \boldsymbol{N}=0 .
$$

From Eq. (57. b) and the condition that the vectors $\overline{\boldsymbol{e}}_{i}$ are linearly independent, we have

$$
T_{, 1}+\bar{\varkappa}_{2} M_{3}-\bar{\varkappa}_{3} M_{2}=0, \quad q_{2}=-M_{3,1}+\bar{\varkappa}_{2} T-\bar{\varkappa}_{1} M_{2}, \quad q_{3}=M_{2,1}+\bar{\varkappa}_{3} T-\bar{\varkappa}_{1} M_{3} .
$$

Equations (58) denote the conditions for equilibrium of moments about the triad $\overline{\boldsymbol{e}}_{i}$. Using Eqs. (57.a), (58. b) and (58. c), we obtain the following conditions for equilibrium of forces in the directions of the triad $e_{i}$ :

$N_{, 1}^{1}+\varkappa_{2} N^{3}-\varkappa_{3} N^{2}=0, \quad N_{, 1}^{2}-\varkappa_{1} N^{3}+\varkappa_{3} N^{1}=0, \quad N_{, 1}^{3}+\varkappa_{1} N^{2}-\varkappa_{2} N_{1}=0$

where

$$
\begin{aligned}
& N^{1}=N-\left(-M_{y, 1}-\varkappa_{1} M_{x}+\varkappa_{2} T\right) u^{2}\left\|_{1}-\left(M_{x, 1}-\varkappa_{1} M_{y}+\varkappa_{3} T\right) u^{3}\right\|_{1}, \\
& N^{2}=N u^{2}\left\|_{1}-M_{y, 1}-T\left(u^{3} \|_{1}\right)_{1}-\varkappa_{1}\left(M_{x}+T u^{2} \|_{1}\right)+\varkappa_{2}\left(T-M_{x} u^{2} \|_{1}\right)-\varkappa_{3} M_{x} u^{3}\right\|_{1}, \\
& N^{3}=N u^{3}\left\|_{1}+M_{x, 1}+T\left(u^{2} \|_{1}\right)_{1}-\chi_{1}\left(M_{y}+T u^{3} \|_{1}\right)-\varkappa_{2} M_{y} u^{2}\right\|_{1}+\varkappa_{3}\left(T-M_{y} u^{3} \|_{1}\right) .
\end{aligned}
$$

Equation (49.d), according to the assumption (5), is written as

$$
T_{, 1}+\varkappa_{2} M_{y}-\varkappa_{3} M_{x}-M_{x}\left(u^{2} \|_{1}\right)_{1}-M_{y}\left(u^{3} \|_{1}\right)_{1}+\varkappa_{1}\left(M_{x} u^{3}\left\|_{1}-M_{y} u^{2}\right\|_{1}\right)=0 \text {. }
$$

Introducing Eq. (61) into the underlined terms in Eqs. (49. i) and (49. j), we find that the coefficients of stress resultants and moments in the underlined terms consist of the second and higher order terms with respect to displacement components. Therefore, according to the assumption (5), the underlined terms in Eqs. (49. i) and (49.j) can be neglected. When we compare Eqs. (48) with Eqs. (58. a) and (59), and recall that the assumption ( 5 ) holds, it is confirmed that the present equilibrium equations derived from the energy method coincide with those derived from the equilibrium method.

The relationships between $q_{2}, q_{3}$ and $Q_{2}, Q_{3}$ are obtained by

$$
Q_{2}=q_{2} \cos \vartheta-q_{3} \sin \vartheta, Q_{3}=q_{2} \sin \vartheta+q_{3} \cos \vartheta \text {. }
$$

The shear forces $q_{2}$ and $q_{3}$ are given by the conditions for equilibrium of moments. While $Q_{2}$ and $Q_{3}$ are the notations introduced in Eqs. (49) for brevity. However, the physical meaning of $Q_{2}$ and $Q_{3}$ are given from Eqs. (62).

\section{CONCLUSIONS}

We have developed a geometrically nonlinear theory of naturally curved and twisted rods by taking account of the coupling of finite rotations in space. There have been various approaches for evaluating finite rotations. In this paper, the finite rotation vector $\theta$ is utilized as a measure for finite rotations and the new variable $\vartheta$ is introduced as the fourth parameter. It has been stressed that the choice of the fourth parameter concerns the development of a finite displacement theory of rods. The significant advantages of the present approach are that the exact displacement functions are obtained through pure mathematical manipulations, and that the exact twist and curvatures after the deformation are calculated with much less efforts. 


\section{ACKNOWLEDGEMENTS}

The authors would like to thank Dr. T. Okumura, Professor Emeritus of the University of Tokyo and Professor of Tokyo Denki University, for his valuable suggestions, and $\mathrm{Ph}$. D. K. Matsui, Professor of Tokyo Denki University, for reading and criticizing the manuscript. Thanks are also due to Professor W. Pietraszkiewicz of Polska Akademia Nauk for providing his papers. The study presented in this paper is supported in part by the Grant-in Aid for Scientific Research from the Japanese Ministry of Education, Science and Culture.

\section{REFERENCES}

1) Nishino, F. and Fukasawa, Y. : Formulation of Static Behavior of Thin-Walled Curved Beams under Assumptions of Strain Field, Proc. of JSCE, No.247, pp.9 19, 1976.3 (in Japanese).

2) Nishino, F., Kurakata, Y., Hasegawa, A. and Okumura, T. : Thin-Walled Members under Axial Force, Bemding and Torsion, Proc. of JSCE, No.225, pp.1 15, 1974.5 (in Japanese).

3) Watanabe, N., Kano, T. and Usuki, S. : Analysis of Large Torsion of a Thin Walled Curved Beam Based on Displacement Field Theory, Proc. of JSCE, No. 317, pp. 31 45, 1982.1 (in Japanese).

4) Usami, T. and Koh, S. Y. : Large Displacement theory of Thin-Walled Curved Members and its Application to LateralTorsional Buckling Analysis of Circular Arches, Int. J. Solids and Structures, Vol. 16, pp. 71 95, 1980.

5) Maeda, Y. and Hayashi, M. : Finite Displacement Analysis of Space Framed Structures, Proc. of JSCE, No. 253, pp. 13 27, 1976.9 (in Japanese).

6) Kurakata, Y. and Nishino, F. : Finite Displacement Field and Governing Equations of Solid Curved Beams, Proc. of JSCE, No. 317, pp. 15 30, 1982.1 (in Japanese).

7) Nishino, F., Kurakata, Y. and Goto, Y. : Finite Displacement Beam Theory, Proc. of JSCE, No. 237, pp. 11 26, 1975. 5 (in Japanese).

8) Besseling, J.F. : Non-linear theory for Elastic Beams and Rods and its Finite Element Representation, Comput. Meths. Appl. Mech. Engrg. 31 (1982) 205 220.

9) Rosen, A. and Friedmann, P. : The Nonlinear Behavior of Elastic Slender Straight Beams Undergoing Small Strains and Moderate Rotations, ASME J. of Appl. Mech., Vol.46, pp. 161 168, 1979.3.

10) Schroeder, F.H. : Allgemeine Stabtheorie des dünnwandigen räumlich vorgekrümmten und vorgewundenen Träger mit grossen Verformungen, Ingenieur-Archiv, 39. Band. S. 87 103, 1970.

11) Hirashima, M., Iura, M. and Yoda, T. : Finite Displacement Theory of Naturally Curved and Twisted Thin-Walled Members, Proc. of JSCE, No. 292, pp. 13 27, 1979. 12 (in Japanese).

12) Kotoguchi, H., Kubo, M. and Nakai, H. : Study on Geometric Non-linearity of Thin-Welled Horizontally Curved Beams and Application to Lateral Buckling Strength of Curved I-Beams, Proc. of 24 th Symp. on Struct. Engrg., pp. 69 76, 1978. 2 (in Japanese).

13) Shimada, S. : Theoretical Analysis for Elastic Wires, Proc. of JSCE, No.72, pp.1 16, 1961.7 (in Japanese).

14) Panayotounakos, D. E. and Theocaris, P. S. : Large Elastic Deformation in Thin Rods, Ingenieur-Archiv, 51. Band, S. 139 149, 1981.

15) Kurakata, Y. : Finite Displacement Theory of Curved Members with Thin-Walled Open Sections, A Dissertation presented for the degree of Doctor of Engineering in the University of Tokyo, 1976 (in Japanese).

16) Ai, M. and Nishino, F. : A Theoretical Formulation of Thin-Walled Beam Elements within Small Strains under Finite Displacements, Proc. of JSCE, No.318, pp.7 20, 1982.2 (in Japanese).

17) Simmonds, J. G. and Danielson, D. A. : Non-linear Shell Theory with Finite Rotation and Stress-Function Vectors, ASME J. of Appl. Mech., Vol. 39, pp. 1085 1090, 1972. 12.

18) Pietraszkiewicz, W. : Finite Rotations and Lagrangian Description in the Non-linear Theory of Shells, Polish Scientific Publishers, Warszawa, 1979 .

19) Iura, M. and Hirashima, M. : Lagrangian Nonlinear Theory of Thin Elastic Shells with Finite Rotations, Proc. of JSCE, No. 344/ I-1, Structural Eng. /Earthquake Eng., pp. 153 163, 1984.4.

20) Pietraszkiewicz, W. and Badur, J. : Finite Rotations in the Description of Continuum Deformation, Int. J. Engrg. Sci., Vol. 21, No. 9, pp. 1097 1115, 1983.

21) Washizu, K. : Variational Methods in Elasticity and Plasticity, Pergamon Press, 3 rd edition, 1982.

(Received March 13 1985) 